\title{
Fuzzy portfolio optimization with tax, transaction cost and investment amount: a developing country case
}

\author{
Gülcan Petriçli ${ }^{1, *}$, A. Gül Gökay Emel ${ }^{1}$, Tuba Bora Kılınçarslan ${ }^{1}$ \\ ${ }^{1}$ Faculty of Economics and Administrative Sciences, Bursa Uludag University \\ Gorukle Campus, Nilufer, Bursa, 16059, Turkey \\ E-mail: 〈\{gulcanp, ggokay, tubabora\}@uludag.edu.tr〉
}

\begin{abstract}
The socioeconomic or political structures of countries and investment costs play a crucial role in investor decisions, especially in developing countries where the environment is unstable. In this regard, fuzzy models that consider the investment amount and cost may enable making more realistic decisions rather than the deterministic models used in portfolio optimization (PO). Hence, the objective of this paper is to examine the effects of the environment, investment amount and cost on PO in a politically, socially and economically unstable environment. Konno-Yamazaki PO model was fuzzified by adopting fuzzy linear programming (FLP) approaches of Verdegay and Werners for this purpose. Afterward, extended models were created. To do that, investment amount, tax and transaction costs were integrated into the return constraint of the fuzzified models. Mean-Variance Model (MVM) of Markowitz was also used for comparatively interpreting the results of the optimization. Results show that the fuzzified models based on Verdegay and Werners FLP approaches can be suggested as a decision-making tool, respectively for risk-averse and risk-taker investors. The extended models provide much better results compared to the fuzzified models. On the other hand, they are not more successful than the MVM in an unstable environment but the stable environment. The main contributions are considering political, social and economic events in the optimization, comparatively analyzing fuzzified Konno-Yamazaki model with its extended versions and the MVM, investigating the relationship between optimization models and investor types.
\end{abstract}

Keywords: fuzzy linear portfolio optimization, transaction cost, tax, investment amount, mixed integer linear programming

Received: September 27, 2019; accepted: October 16, 2019; available online: December 13, 2019

DOI: $10.17535 /$ crorr.2019.0022

\section{Introduction}

Markowitz developed the MVM to determine the weights that minimize the risk while keeping the return constant at a certain level. Thus, the number of the assets in the portfolio and share of them can be determined easily for different expected returns. However, uncertainties resulting from the various socio-economic, social and political situations may prevent the use of MVM by itself (Please refer to the appendix for MVM of Markowitz). Instead, using fuzzy models considering uncertainty may be beneficial to use since fuzzy numbers are powerful for describing impreciseness or vagueness of numeric quantities in the field of decision-making [20], and the selection of the optimal portfolio belongs to that field [19]. Fuzziness/ fuzzy logic-optimization has been widely used and successfully applied in real-life problems in social sciences. Setting up a fuzzy mathematical model, computation and interpretation of it is less complicated and much understandable, and its outputs much easily applicable than that of many other methodologies.

${ }^{*}$ Corresponding author. 
Moreover, the classical Markowitz model is formulated under an $L_{2}$ risk function. Therefore, computational difficulty related to solving quadratic programming problems with a dense covariance matrix is another issue regarding MVM of Markowitz [12]. On the contrary, there is no need to compute the covariance matrix in linear models such as the Konno-Yamazaki model. Konno-Yamazaki model is formulated under an $L_{1}$ risk function. Therefore solving it is much easier than solving a quadratic problem. Also, controlling the number of assets in the portfolio is an easy task with a control variable in Konno-Yamazaki model comparing to MVM model. Therefore, Konno-Yamazaki PO model was fuzzified with Verdegay and Werners FLP approaches $[32,34]$ in this paper.

Cost and the investment amount is another issue in PO. Many existing studies have shown that transaction costs can significantly affect investment behavior. There are several kinds of transaction costs like proportional, fixed, linear or non-linear [20]. Omitting them or deducting the cost from the portfolio return may result in inefficient portfolios. In other words, costs can erode the gains from a trading strategy [25]. Hence, fuzzified Konno-Yamazaki models were extended with the inclusion of tax and transaction costs for different investment amounts in this study. The data were collected from the capital market Istanbul Stock Exchange (Borsa Istanbul-BIST) and a state bank in Turkey for the period between 01.01.2013 and 30.03.2014.

The organization of this paper is as follows. The relationship between PO and FLP was discussed in the preliminaries section. In this scope, the approaches of Konno-Yamazaki [13], Verdegay [32] and Werners [34] were explained. Then the effects of costs in the PO were discussed, and the extended models considering costs were explained. It is followed by the data, application, results, conclusion and appendix section.

\section{Preliminaries}

The primary objective of portfolio management is to create effective portfolios about the risk and return balance. The determination of an effective portfolio can be done via quadratic programming by calculating the expected return and the variance-covariance values of assets. However, this method does not consider the uncertainty level of decision-makers. That is due to the financial markets which cause immediate decision changes in investors influenced by uncontrollable events such as natural disasters, corruption and strikes. This change is entirely shaped by the perception, experience and insufficient knowledge of investors. Hence using fuzzy methods may be beneficial as it is a practical approach when there is insufficient information regarding the event. Indeed, fuzzy PO methods ensure a certain level of satisfaction and may provide more realistic results than deterministic models [2, 33].

Almost hundreds of studies have been carried out on fuzzy PO in the past 30 years. One of the most important ones was the study carried out by [26]. In that study, a dynamic PO model was developed by fuzzifying the uncertainty of the future prices and risks of the assets. Ramaswamy [28] developed fuzzy multi-criteria linear programming models for several rates of return levels. Fuzziness was applied to the subjective decisions of investors for an uncertain period via trapezoidal membership function. The objective was the fuzzified utility maximization. Tanaka and Guo [30] used possibility distributions instead of average variance for representing the knowledge of decision-makers. They partially rejected the assumption of Markowitz regarding the effectiveness of the past data in the future by integrating it with the knowledge and judgments of decision-makers. They generated an exponential possibility distribution for each decision-maker and integrated them into one. According to [17], PO models should have considered the nature of the data, risk and return expectations. In this regard, they examined several approaches regarding the data uncertainty and re-optimized the infeasible models by using duality theory and fuzzy programming. Another fuzzy PO model that was based on MVM was developed by Watada [33]. The satisfaction level for the expected return and risk was defined by a logistic membership function. 
In PO, the Konno-Yamazaki Linear Programming model provides more effective results at large scale problems in comparison to the Markowitz model. That is why it is one of the most frequently used deterministic methods in PO [8, 27]. The objective of the Konno-Yamazaki model is to determine the assets with the lowest deviation at any return level. In other words, this is a method that takes average absolute deviation as a reference $[8,27]$, and focuses on assets of which the average return is equivalent to the desired return level $[12,13]$. The model is solved repetitively for each expected return since it is deterministic and thus provides instantaneous results to the investor. The following model is due to Konno and Yamazaki [12].

Model 1(M1):

$$
\min _{y_{t}} \frac{1}{T} \sum_{t}^{T} y_{t}
$$

subject to:

$$
\begin{aligned}
y_{t}+\sum_{j=1}^{n}\left[\left(r_{t j}-r_{j}\right) x_{j}\right] & \geq 0, t=1,2, \ldots, T, \\
y_{t}-\sum_{j=1}^{n}\left[\left(r_{t j}-r_{j}\right) x_{j}\right] & \geq 0, t=1,2, \ldots, T, \\
\sum_{j=1}^{n}\left(r_{j} x_{j}\right) & \geq \rho M_{0}, \\
\sum_{j=1}^{n} x_{j} & =M_{0}=1, \\
y_{t} & \geq 0, t=1, \ldots, T .
\end{aligned}
$$

In the Konno-Yamazaki model, $T$ is the number of periods, $j$ is the number of assets, $\rho$ is the rate of expected return, $r_{j}$ is the average rate of return of the $j^{t h}$ asset, $x_{j}$ is the share of the jth asset, $t$ is the $t^{t h}$ period, $y_{t}$ is the assistant variable, $r_{t j}$ is the real return of the $j^{t h}$ asset at the $t^{t h}$ period and $M_{0}$ is the total investment amount. $r_{t j}-r_{j}$ is the difference (deviation) between the real rate of return of the $j^{\text {th }}$ asset in the tth period and the average rate of return of the same asset for the total $T$ period. This difference represents a risk. Assets with a minimum risk of zero are determined for each t period with the Eq. (2). Therefore, assets with negative deviation are eliminated with it. Assets with the smallest risk are determined with the Eq. (3). Assets of which average risk (return ratio) is equal to or greater than the total expected return are determined with the Eq. (4). The sum of the investment amounts is equated with the Eq. (5). The objective in the model minimizes the deviation from the total expected return $\rho$. In other words, it is the determination of the $x_{j}$ 's of which risk is minimum and average return is equal to or greater than the expected return by minimizing the $y_{t}$ assistant variable at period $t$.

It is known that the optimal solution of a linear programming model only depends on the constraints, and much of the information present in the data is ignored. The use of probabilistic distributions allows to account for more information, but it may be impossible to collect enough data for determining the probability distribution in emerging stock markets without enough historical data such as in China [20] and similar as in Turkey. With the introduction of the concept of fuzzy sets in the seminal paper of L. A. Zadeh [38], we have an alternative and powerful way of modeling data information without using stochastic concepts [29]. Fuzzy logic can express uncertain knowledge and makes it suitable for representing the inherently uncertain nature of PO problem [19]. 

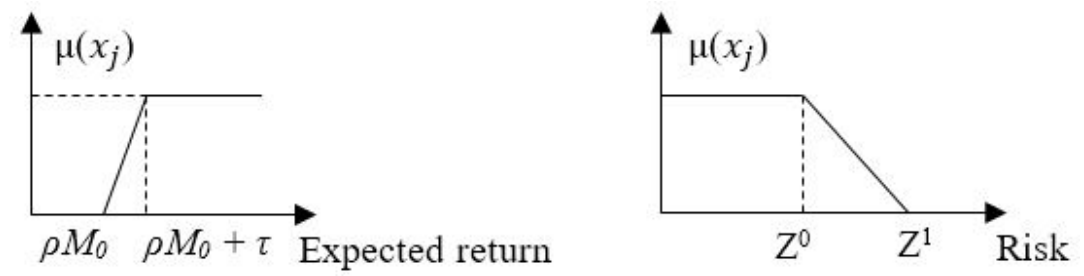

Figure 1: The fuzzy trapezoidal membership function of (a) expected return and (b) risk

The fundamental difference between a linear programming and an FLP model is the resource constraints or/and the objective function in an FLP model being in the form of approximate inequalities. Approximateness can be provided by membership functions. When the demands of investors and the nature of the problem are considered; trapezoidal and logistic membership functions can be used $[18,33]$. There are several approaches available in FLP. For example, the objective function and criteria in Zimmermann approach are fuzzified by a tolerance interval, which is initially given [16, 39]. Similarly, an interval is used for the same purpose also in Werners approach, but it is calculated with specific formulations $[16,34,11]$. On the other hand, in the Verdegay approach, only the criteria are fuzzified, and the model is solved repetitively for different satisfaction levels $[32,12]$. In this study, only Verdegay and Werners FLP approaches were adopted as there were no real decision-makers to determine a tolerance interval for Zimmermann method. [16]:

The general model of linear programming with fuzzy constraints is formulated as follows

Model 2:

$\max _{x} c x$

subject to:

$$
\begin{aligned}
(A x)_{i} & \lesssim b_{i}, i=1, \ldots, m \\
x & \geq 0
\end{aligned}
$$

where $\lesssim$ is fuzzy less than or equal to and $b_{i}$ is in $\left[b_{i}, b_{i}+p_{i}\right] \forall i$ with a given tolerance $p_{i}$. The value of the $p_{i}$ or in other words upper and lower limits of the tolerance, so the right-hand side of Eq. (8), varies depending on a $\theta$ which is in $[0,1]$. Therefore, $(A x)_{i} \lesssim b_{i}$ is equivalent to $(A x)_{i} \lesssim b_{i}+\theta p_{i}$. Hence, as in the paper of Kocadağliand Cinemre [11], if the rate of return (expected return) $\rho$ in Eq. (4) is assumed to be a fuzzy number since its value is between $[0,1]$, the model becomes a fuzzy model. If $\tau$ denotes the tolerance variable of the expected return, $\rho$ denotes the average of the expected returns and $\rho_{\max }$ represents the maximum of it, then the tolerance of the expected return is calculated as $\tau=\rho_{\max }-\rho$. The sum of $\rho \cdot M_{0}+\tau$ is accepted as the upper limit of the expected return. Therefore, the membership function of the constraint can be arranged as below in Eq. (9) and Figure 1(a) as it is monotonically increasing.

$$
\mu(x)=\left\{\begin{array}{cl}
0, & \sum_{j=1}^{n}\left[\left(r_{t j}-r_{j}\right) x_{j}\right]<\rho M_{0} \\
\frac{\left[\sum_{j=1}^{n}\left[\left(r_{t j}-r_{j}\right) x_{j}\right]-\rho M_{0}\right]}{\tau}, & \rho M_{0} \leq \sum_{j=1}^{n}\left[\left(r_{t j}-r_{j}\right) x_{j}\right] \leq \rho M_{0}+\tau \\
1, & \sum_{j=1}^{n}\left[\left(r_{t j}-r_{j}\right) x_{j}\right]>\rho M_{0}+\tau
\end{array}\right.
$$

Note that the membership function $\mu(x)$ does not start from zero. The reason for this is that an investor expects a return equal to or higher than the average return of the portfolio. Thus, the minimum expected return of the investor does not start from zero but a positive value on the $x$-axis. As seen in Eq. (9) and Figure 1(a), functions are continuous and monotonic. 
Therefore, the model, in general, is represented as below [16].

Model 3:

$$
\min Z
$$

subject to

$$
x \in X_{\alpha}
$$

where $X_{\alpha}=\left\{x \mid \forall i, \mu_{i}(x) \geq \alpha, x \geq 0\right\}$, for eqach $\alpha$-level cut (satisfaction level) $\alpha \in[0,1]$. When Eq. (9) is applied to model 3 and Eq. (4), M1 is rearranged as below.

Model 4 (M4):

$$
\min _{y_{t}} \frac{1}{T} \sum_{t}^{T} y_{t},
$$

subject to:

$$
\begin{gathered}
y_{t}+\sum_{j=1}^{n}\left[\left(r_{t j}-r_{j}\right) x_{j}\right] \geq 0, t=1,2, \ldots, T, \\
y_{t}-\sum_{j=1}^{n}\left[\left(r_{t j}-r_{j}\right) x_{j}\right] \geq 0, t=1,2, \ldots, T, \\
\sum_{j=1}^{n}\left(r_{j} x_{j}\right) \geq \rho M_{0}+\alpha \tau, \alpha \in[0,1] \\
\sum_{j=1}^{n} x_{j}=M_{0}=1, \\
y_{t} \geq 0, t=1, \ldots, T .
\end{gathered}
$$

In the Verdegay approach, only the return constraint has been fuzzified, and the objective function has been bound indirectly to the fuzzy return constraint via a variable, $y_{t}$. Calculations should be carried out repeatedly for different satisfaction levels. However, because of the fuzzy constraint, Eq. (15), in M4, the objective function should be fuzzified as well [16]. Moreover, the objective in real life is to determine an $\alpha$, which provides optimum satisfaction for different risk and return combinations simultaneously. This determination can be made via the Werners approach [16].

In Werners approach, $\alpha$ is tried to be maximized. Werners stated that the objective function of Model 2 should be fuzzy because of fuzzy constraints. Let us assume that tolerance $p_{i}$ for the fuzzy constraints is given again. Then Model 2 is repetitively solved for the lower and upper limits of $\mathrm{Eq}(8)$ as below:

$$
\begin{gathered}
Z^{0}=\max _{x} c x \text { subject to }(A x)_{i} \lesssim b_{i}, \forall i, x \geq 0 \\
Z^{1}=\max _{x} c x \text { subject to }(A x)_{i} \lesssim b_{i}+p_{i}, \forall i, x \geq 0
\end{gathered}
$$

Then a membership function for the objective (maximization) is created as in Figure 1(b). As it is a continuously decreasing linear membership function, it is formulized as below in Eq. (18).

$$
\mu(x)=\left\{\begin{array}{cl}
1, & Z<Z^{0} \\
1-\frac{\left(Z-Z^{0}\right)}{\left(Z^{1}-Z^{0}\right)}, & Z^{0} \leq Z \leq Z^{1} \\
0, & Z>Z^{1}
\end{array}\right.
$$

In this respect, M4 is solved repetitively by taking satisfaction level $\alpha=0$ and $\alpha=1$. Thus obtaining a risk value $Z^{0}$ for $0 \%$ satisfaction and a risk value $Z^{1}$ for $100 \%$ satisfaction. 
The max-min operator can be used to obtain an optimal decision, and model 2 can be solved by using $\max _{x \geq 0} \alpha$, where $\alpha=\min \left[\mu_{0}(x), \mu_{1}(x), \ldots, \mu_{m}(x)\right]$. That is

Model 5:

$\max \alpha$

subject to:

$$
\begin{gathered}
\mu_{0}(x) \geq \alpha \\
\mu_{i}(x) \geq \alpha, \forall i \\
\alpha \in[0,1] x \geq 0
\end{gathered}
$$

Therefore Eq.(13), is applied on model 5, and M4 is rearranged as below Model 6 (M6):

$\max \alpha$

subject to:

$$
\begin{aligned}
y_{t}+\sum_{j=1}^{n}\left[\left(r_{t j}-r_{j}\right) x_{j}\right] & \geq 0, t=1,2, \ldots, T, \\
y_{t}-\sum_{j=1}^{n}\left[\left(r_{t j}-r_{j}\right) x_{j}\right] & \geq 0, t=1,2, \ldots, T, \\
\sum_{j=1}^{n}\left(r_{j} x_{j}\right) & \geq \rho M_{0}+\alpha \tau, \alpha \in[0,1], \\
\sum_{j=1}^{n} x_{j} & =M_{0}=1, \\
\frac{1}{T} \sum_{t=1}^{T}\left(y_{t}\right)+\alpha\left(Z^{1}-Z^{0}\right) & \leq Z^{1} \\
y_{t} & \geq 0, t=1, \ldots, T .
\end{aligned}
$$

The risk value $Z$ corresponds to the optimized $\alpha$ value and is in the interval of $Z^{1} \leq Z \leq Z^{0}$. However, the model does not provide the risk value directly. It should be calculated by using the graph in Fig. 1(b).

\section{Costs in portfolio optimization}

The use of fuzzy models by themselves does not sufficiently ensure the convergence of the optimization results to reality. To explain, investors seek to create portfolios that provide minimum risk or maximum satisfaction by increasing their net incomes. An optimization model that does not include costs results in the generation of non-effective portfolios [22], and optimization results and portfolio performance are significantly affected [1].

The most frequently encountered portfolio costs are transaction, commission, management, performance (liquidity) costs and taxes. Transaction costs may be constant (fixed) or proportional $[10,21]$. Fixed transaction costs increase with the diversification as the fixed costs vary with the number of assets in the portfolio. Hence the number of assets in the portfolio might decrease with the optimization [22]. On the contrary, proportional transaction costs depend on the investment amount. Contrary to fixed costs, proportional transaction costs encourage the 
variety of tools included in the portfolio. Moreover, their shares decrease when the investment amount increases [15, 22, 31].

In the literature, it has been mentioned that transaction costs are significant for portfolio optimization [7], and such costs are added indirectly only by subtracting the cost from portfolio return due to the calculation load and the complex structure of the model. As was stated by [22], transaction costs have been included in the model as a constraint only in several studies, such as $[35,37]$. Whereas in practice, transaction costs may become meaningless concerning large investment amounts. Therefore, there is no drawback in neglecting these costs [14]. However, transaction costs are significant for cases when portfolio return is low and also for investors who make frequent purchases/sales [15]. Moreover, in recent studies like [25], the importance of transaction cost and investment amount on the portfolio has been comparatively examined by using different methodologies like robust PO and Bayesian PO. According to the authors, investment amount and cost interacts with each other. For example, small trades do not impact the market price, and the transaction cost is assumed to be proportional to the amount traded. Larger trades impact the market price, and it is assumed that it results in quadratic transaction cost $[24,25]$.

The addition of real constraints to such mathematical models makes the already complex model even more complicated. The cost function may be linear, piecewise linear, constant, piecewise constant linear or concave/convex nonlinear. For example, in the case of proportional transaction costs, the linearity of the model may be disrupted because this kinds of cost structures may require the transformation of the linear model into a piecewise linear structure [9] and the addition of integer or (0-1) binary variables [22]. As a result, the model becomes a mixed-integer linear programming model. It is stated that the optimization is much easier when the transaction costs are linear or constant in comparison with other cases [21, 31]. According to Konno and Yamamoto [15], the most frequently encountered transaction cost functions are piecewise linear concave and piecewise constant linear. Therefore, the inclusion of this kind of cost variables requires the use of binary variables in the optimization.

According to Mansini et al. [22], there are two types of models in portfolio optimization problems that have real futures, namely relative and absolute. If a model has decision variables in shares or percentages, the model is called the relative model and the variables relative. However, a model may have real futures in absolute values as well. In this case, the model is called the absolute model and the variables absolute.

In this paper, the fixed cost was not considered because no information could be obtained from the state bank. In such a case, a hypothetic constraint could have been set. However, we prefer not to set it because all the data and cost information used in the models were real. Nevertheless, two equations Eq. $(35,36)$ were proposed for the inclusion of the fixed cost later in the appendix in order to partially overcome this limitation of the study. The models in this paper become mixed-integer linear programming models with the inclusion of the proposed equations, Eq. (35, 36).

For the inclusion of the tax (BITT - Banking and Insurance Transactions Tax) and transaction cost, variables of which values were real futures were generated, but it was impossible to include them into M4 and M6 as they were relative models. Hence, the return constraint Eq. (15) was transformed into an absolute structure by multiplying the right and left-hand sides of it by the investment amount $M_{R}$. After that, the $\operatorname{tax} C_{T X}$ and the transaction cost $C_{T R}$ were subtracted from the left-hand side of it. However, $C_{T R}$ is a function depending on the investment amount, and can be determined before the optimization. Hence, it should be transferred to the right side of the constraint. On the other hand, $C_{T X}$ is a function that depends on the portfolio return so the investment amount and the share of each asset. Since the shares are indefinite, it is unknown. Hence, it should be on the left side. Finally, new form of Eq. (15) is as below: 


$$
\begin{array}{r}
\sum_{j=1}^{n}\left(r_{j} x_{j}\right) \geq \rho M_{0}+\alpha \tau, \alpha \in[0,1] \\
M_{R} \sum_{j=1}^{n}\left(r_{j} x_{j}\right)-C_{T R}-C_{T X} \geq M_{R}\left(\rho M_{0}+\alpha \tau\right) \\
M_{R} \sum_{j=1}^{n}\left(r_{j} x_{j}\right)-C_{T X} \geq M_{R}\left(\rho M_{0}+\alpha \tau\right)+C_{T R}, \alpha \in[0,1]
\end{array}
$$

As stated previously, the investment amount directly affects the portfolio structure $[15$, $22,31]$. The number of tools increases, and shares decrease especially when the investment amount increases. Even though the investment amount was not considered as a variable to be optimized in this paper, optimizations were done for different given investment amounts to investigate its effects on the portfolios. Then a set of constraints Eq. (37-43) was proposed for the purpose of optimizing the investment amount in the appendix. The models in this paper become mixed-integer quadratic programming models with the inclusion of the proposed equations, Eq. (37-43).

\section{Data}

Turkey was among the countries where uncertainty was more considerable than in developed countries. Some political and social events in Turkey had created anxiety in foreign investors and IMF $[3,5]$ in 2013 and 2014. It was also stated in an article by CNN titled "Is instability the 'new normal' for Turkey?" [4]. However, according to some other sources, Turkey was also on a positive trajectory [36] during that time. Therefore, it may have been difficult for investors to make decisions in such fuzzy environments where social and political uncertainties abounded together with different interpretations. Hence, Turkey was considered to be a wellsuited country for examining the effects of the uncertain environment, investment amount and costs on the optimum portfolio.

The effects of the environment were investigated by dividing the investment period into sub-periods of which starting and ending dates were either social, political or financial events. Firstly, critical events in 2013 and 2014 were determined. Then the whole period in question was divided into five sub-periods. Afterward, continuity of the 30 assets between periods was checked. It was determined that the number of assets in each period differs because some assets were delisted and trading for some others was suspended temporarily between the sub-periods. For example, 32 assets in the first and second periods, 33 assets in the third period, and 31 assets in the fourth and fifth periods were continuous. Then, daily data for the stocks and closing prices for gold, US Dollar and Euro parities was collected from BIST. Before the application, raw data were standardized and graphically visualized, and major breaking points were determined for examining if heuristically determined periods fit the reality. As seen in Figure 2 , the whole period had five sub-periods of which starting and ending dates complied with the abovementioned dates. The first period (01.01.2013 - 27.05.2013) was the time interval before the Gezi Park protests and FED declaration regarding the reduction of monetary expansion (1st FED declaration). Most probably, as a result of these events, international funds exited Turkey by consuming foreign currency in the country during that period. The second period (28.05.2013 - 21.08.2013) started with Gezi Park protests and ended with the $2^{\text {nd }}$ FED declaration in which no statement was made regarding when the expansion would be completed. In experts' opinion, the Turkish market had been affected negatively by this uncertainty. The third period $(22.08 .2013-17.09 .2013)$ was the time interval between the $2^{\text {nd }}$ FED declaration 


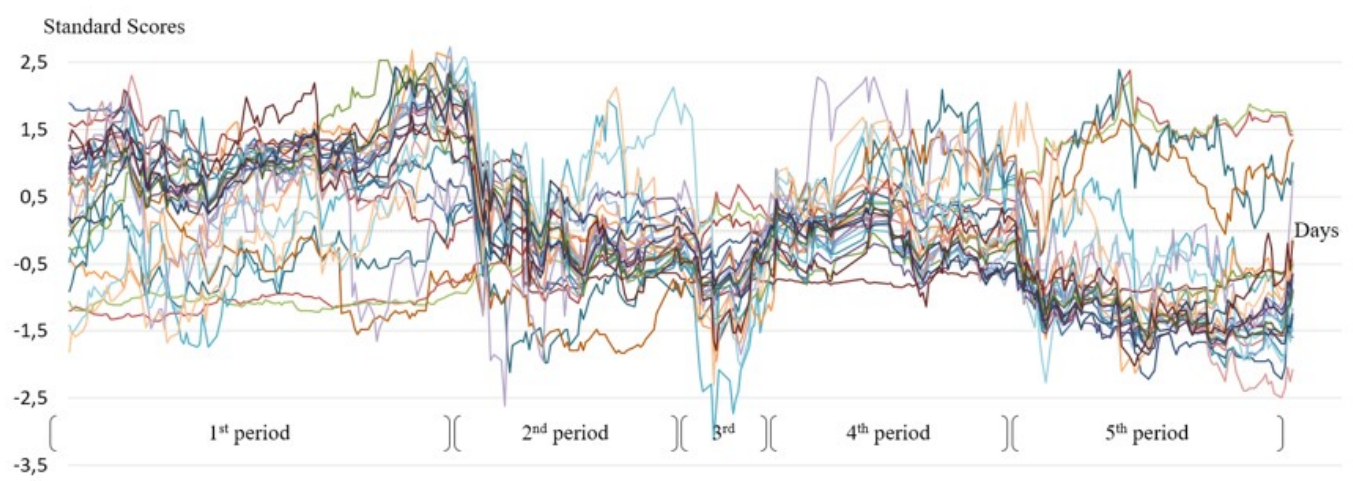

Figure 2: Graphical visualization of the standardized data
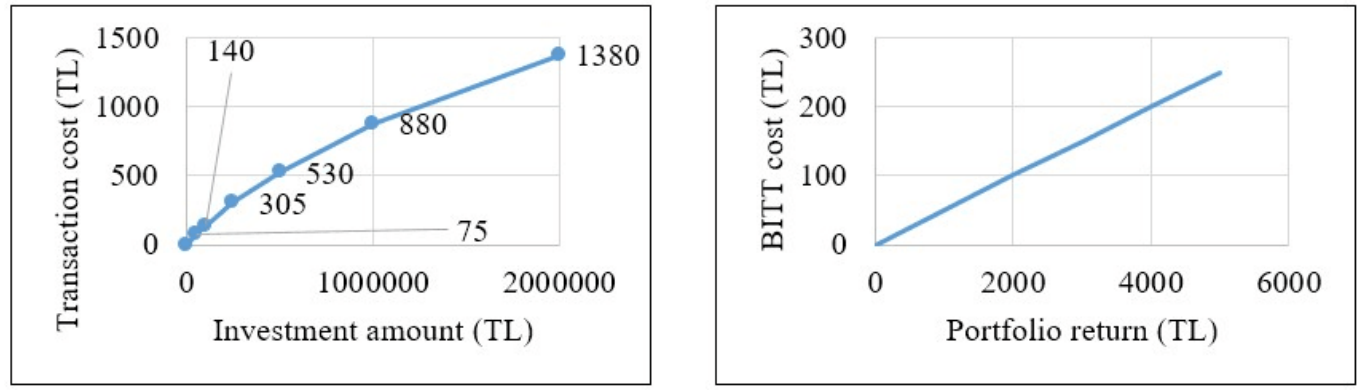

Figure 3: (a) Transaction cost and (b) tax function

and the $3^{\text {rd }}$ FED declaration stating that monetary expansion would not be reduced. It was put forth that the markets experienced relief with the declaration. The fourth period (18.09.2013 - 16.12.2013) started with the $3^{\text {rd }}$ FED declaration and ended with the December 17 investigations, which was a political crisis in Turkey. The last period (17.12.2013 - 30.03.2014) was the time interval started with the investigation in Turkey to the local elections. In the end, the cost data were obtained from a state bank in Turkey.

\section{Application}

Transaction costs are calculated over the capital invested, whereas BITT is reflected in the investor over the acquired return. The cost functions should be determined based on the data obtained from a state bank in Turkey to integrate transaction and tax costs to the Verdegay and Werners models. BITT rate is 0.05 for any amount of return. Hence its function is constant. On the contrary, the transaction cost rate differs for different investment amounts in Turkish Lira (TL): 0.0015 for $\left(0<M_{R} \leq 50,000\right)$, 0.0013 for $\left(50,000<M_{R} \leq 100,000\right)$, 0.0011 for $\left(100,000<M_{R} \leq 250,000\right), 0.0009$ for $\left(250,000<M_{R} \leq 500,000\right)$, 0.0007 for $\left(500,000<M_{R} \leq 1,000,000\right)$ and 0.0005 for $\left(100,000,000<M_{R}\right)$. Therefore, it is variant, and its function is piecewise constant linear. After obtaining the rate data, their functions have been determined, as in Figure 3(a) via the line equation with slope and one point where $f\left(b_{n}\right)$ represents the function of each line segment, and $\mathrm{n}$ represents the number of divides. For example, there are seven divides and six line segments of which functions are $f\left(b_{1}\right)=$ $0.0015 M_{R}, f\left(b_{2}\right)=0.0013 M_{R}, f\left(b_{3}\right)=0.0011 M_{R}, f\left(b_{4}\right)=0.0009 M_{R}, f\left(b_{5}\right)=0.0007 M_{R}$ and $f\left(b_{6}\right)=0.0005 M_{R}$ respectively.

Afterward, an investment amount on each line segment was determined arbitrarily, and 
their transaction costs were calculated as follows: $C_{(T R(40,000))}=60 T L, C_{(T R(90,000))}=127 T L$, $C_{(T R(200,000))}=250 T L, C_{(T R(400,000))}=440 T L, C_{(T R(900,000))}=810 T L, C_{(T R(2,000,000))}=$ $1380 T L$. As an example, transaction cost for $900.000 T L$ was $C_{(T R(900,000))}=530+(0.007(900,000-$ $500,000))=810 T L$.

As was explained previously, BITT is calculated over the portfolio return, and it cannot be determined at this point since it is not for sure how much return of the portfolio will be. Thus, symbolic data were used for BITT cost and Figure 3(b) was obtained. The goal here was to determine the tax function in TL.

As can be seen from Figure 3(b), the cost function of the tax has a linearly increasing trend hence it can be represented with $C_{T X}=0.05 M_{R} \sum_{j=4}^{n}\left(r_{j} x_{j}\right)$. The subscript $j$, which represents the asset number, starts from four due to a particular instance of the data used in the study. As was stated previously, the portfolio pool not only contains the stocks in BIST30 but also gold, Euro and US Dollar, and these assets are the first three instruments for which tax costs have not been reflected upon. Hence, the first value of the $\mathrm{j}$ was assigned four, and it became necessary to rearrange the Eq. (30) as follows.

$$
M_{R} \sum_{j=1}^{n=3}\left(r_{j} x_{j}\right)+0.95 M_{R} \sum_{j=4}^{n}\left(r_{j} x_{j}\right) \geq M_{R}\left(\rho M_{0}+\alpha \tau\right)+C_{T R}, \alpha \in[0,1]
$$

\section{Results}

Firstly, the optimization was done with M4 and M6 for each investment period and each investment amount. Then Eq. (15) was replaced by the Eq. (31) in M4 and M6 in order to obtain the extended M4 (e-M4) and extended M6 (e-M6), and the optimization repeated with the extended models. Finally, MVM of Markowitz was used for each expected return obtained from the extended models in order to interpret the results comparatively.

Rate of expected return, $\rho$ for each period were just above zero, $0.000924833,-0.003413221$, $0.004737533,0.000578897$ and -0.000414058 respectively. That means there were several assets of which average rate of return, $r_{j}$ were negative in each period. As a result, there were only $14,4,6,9$ and 8 assets in the optimized portfolios for each investment period. Satisfaction for each period was calculated respectively as $0,0.2087,0,0.0137$ and 0.2602 with M4. The risk levels corresponding to the satisfactions were determined respectively as 0.0021, 0.0335, 0.0004, 0.0023 and 0.0390 .

$Z^{1}$ risk values were determined as infeasible at $\alpha=1$ level in the second and fifth period in M6. Thus, optimization could not be carried out for these periods with that model. The satisfaction levels obtained for the first, third and fourth periods were $0.6660,0.6174$ and 0.6136 , and there were 9, 2 and 7 assets in their corresponding portfolios. Since M6 cannot provide satisfaction level and risk simultaneously, risk values of that periods were determined by using the graph in Figure 1(b). They were 0.0072, 0.0019 and 0.0092.

When all investment periods are examined based on the results mentioned above, M6 provides a higher satisfaction level in comparison with M4. It gives this by slightly increasing the risk. Another finding is that the portfolios generated via M4 are more extensive in comparison with those created via M6. It means that M4 that adopts the Verdegay approach provides a more varied portfolio. This variety may be because the objective function minimizes the risk by increasing the number of assets. Another finding is that M6 has prevented the decisionmakers from investing during the second and fifth periods. These periods are the time when the Gezi Park events and December 17 Investigations drifted the country towards uncertainty. The average returns of these periods are negative as expected.

When optimization was carried out via e-M4 and e-M6, all results of e-M6 were infeasible. In other words, e-M6 prevented decision-makers from making investments. On the other hand, 
e-M4 provided considerable increases in satisfaction level by making slight increases in the risk as seen in Table 1. It can also be observed that the portfolios obtained in this model were smaller in comparison with those obtained via M4, that the tools included in the portfolio varied about type and proportion, and that the portfolio structure changed with different investment amounts. Another observation is that the portfolios and optimization results for the second and the fifth period were the same for both M4 and e-M4. The reason for this is that the average return of the assets in this period is negative. Also, portfolios comprised of only a single instrument were generated for some investment amounts during the first, fourth and fifth periods.

\begin{tabular}{|c|c|c|c|c|c|c|c|c|c|}
\hline \multirow{2}{*}{ Period } & \multirow{2}{*}{$\begin{array}{c}\text { Invest. } \\
\text { Amount } \\
\text { (x1000) } \\
\text { TL }\end{array}$} & \multirow{2}{*}{$\alpha$} & \multirow{2}{*}{ Risk } & \multicolumn{2}{|c|}{ Num. of Assets } & \multirow{2}{*}{ Return } & \multicolumn{3}{|c|}{ PCR } \\
\hline & & & & e-M4 & MVM & & $\mathrm{M}^{*}$ & e-M4 & MVM $^{*}$ \\
\hline \multirow{6}{*}{1} & 40 & \multirow{2}{*}{0} & 0.0066 & \multirow{2}{*}{8} & \multirow{2}{*}{8} & 0.0025 & -0.3970 & 0.5699 & 0.5701 \\
\hline & 90 & & 0.0059 & & & 0.0023 & -0.3065 & 0.6555 & 0.6559 \\
\hline & 200 & 0.5573 & \multirow{2}{*}{0.0176} & \multirow{2}{*}{1} & \multirow{2}{*}{2} & \multirow{2}{*}{0.0039} & -0.1842 & 1.9746 & 1.9746 \\
\hline & 400 & 0.6528 & & & & & -0.0095 & 2.5194 & 2.5194 \\
\hline & 900 & \multirow{2}{*}{0} & 0.0040 & \multirow{2}{*}{8} & 10 & 0.0013 & 0.2604 & 0.7213 & 0.5004 \\
\hline & 2000 & & 0.0033 & & 11 & 0.0011 & 0.7327 & 1.1122 & 0.7812 \\
\hline \multirow[t]{6}{*}{2} & 40 & \multirow{6}{*}{0} & \multirow{6}{*}{0.0035} & \multirow{6}{*}{4} & \multirow{6}{*}{6} & \multirow{6}{*}{0.0010} & -0.2994 & -0.2994 & -0.2963 \\
\hline & 90 & & & & & & -0.1916 & -0.1916 & -0.1913 \\
\hline & 200 & & & & & & -0.0447 & -0.0447 & -0.0443 \\
\hline & 400 & & & & & & 0.1674 & 0.1674 & 0.1681 \\
\hline & 900 & & & & & & 0.5007 & 0.5007 & 0.5018 \\
\hline & 2000 & & & & & & 1.1004 & 1.1004 & 1.1025 \\
\hline \multirow{6}{*}{3} & 40 & \multirow{6}{*}{0} & 0.0065 & 5 & 6 & 0.0065 & 1.7562 & 2.6136 & 2.6083 \\
\hline & 90 & & 0.0062 & 6 & 7 & 0.0064 & 2.1191 & 2.9689 & 2.9634 \\
\hline & 200 & & 0.0059 & 6 & 6 & 0.0061 & 2.5921 & 3.4056 & 3.3993 \\
\hline & 400 & & 0.0056 & \multirow{2}{*}{7} & \multirow{2}{*}{7} & 0.0059 & 3.2342 & 3.9861 & 3.9767 \\
\hline & 900 & & 0.0052 & & & 0.0057 & 4.1557 & 4.8906 & 4.8780 \\
\hline & 2000 & & 0.0055 & 6 & 7 & 0.0056 & 5.5900 & 6.3429 & 6.3243 \\
\hline & 40 & 0 & 0.0138 & 3 & 7 & 0.0022 & -0.5958 & 0.3597 & 0.3670 \\
\hline & 90 & 0.4499 & & & & & -0.5345 & 1.2139 & 1.2139 \\
\hline & 200 & 0.5252 & & & & & -0.4512 & 1.5648 & 1.5648 \\
\hline 4 & 400 & 0.6004 & 0.0203 & 1 & 1 & 0.0032 & -0.3316 & 2.0479 & 2.0479 \\
\hline & 900 & 0.6757 & & & & & -0.1453 & 2.7552 & 2.7552 \\
\hline & 2000 & 0.7510 & & & & & 0.1849 & 3.8901 & 3.8901 \\
\hline & 40 & 0 & 0.0052 & 6 & 8 & 0.0011 & -0.4926 & -0.2733 & -0.2752 \\
\hline & 90 & 0.4820 & 00226 & 1 & 1 & 0.0023 & -0.4149 & 0.6382 & 0.6382 \\
\hline & 200 & 0.5552 & 0.0226 & 1 & 1 & 0.0023 & -0.3091 & 0.9077 & 0.9077 \\
\hline 5 & 400 & 0.0812 & & & & & -0.1566 & -0.2130 & -0.2128 \\
\hline & 900 & 0.1544 & 0.0396 & 8 & 9 & 0.0007 & 0.0823 & 0.0108 & 0.0111 \\
\hline & 2000 & 0.2275 & & & & & 0.5100 & 0.4123 & 0.4128 \\
\hline
\end{tabular}

Table 1: Results of the optimization carried out via M4, e-M4 and MVM ( ${ }^{*}$ Costs are reflected after the optimization by deducting them from the portfolio return.)

As explained previously, e-M4 does consider transaction cost and tax for different investment amounts, whereas M4 does not. Expectedly, e-M4 provided higher PCR values, while the reflection of the transaction cost and tax upon the optimized M4 models caused lower or negative PCR, as seen in Table 1.

Finally, MVM of Markowitz was used for the optimization. The optimization was done for only return values of the portfolios generated with e-M4 since the optimization results of e-M6 were all infeasible. Portfolios obtained with MVM of Markowitz were more extensive in 


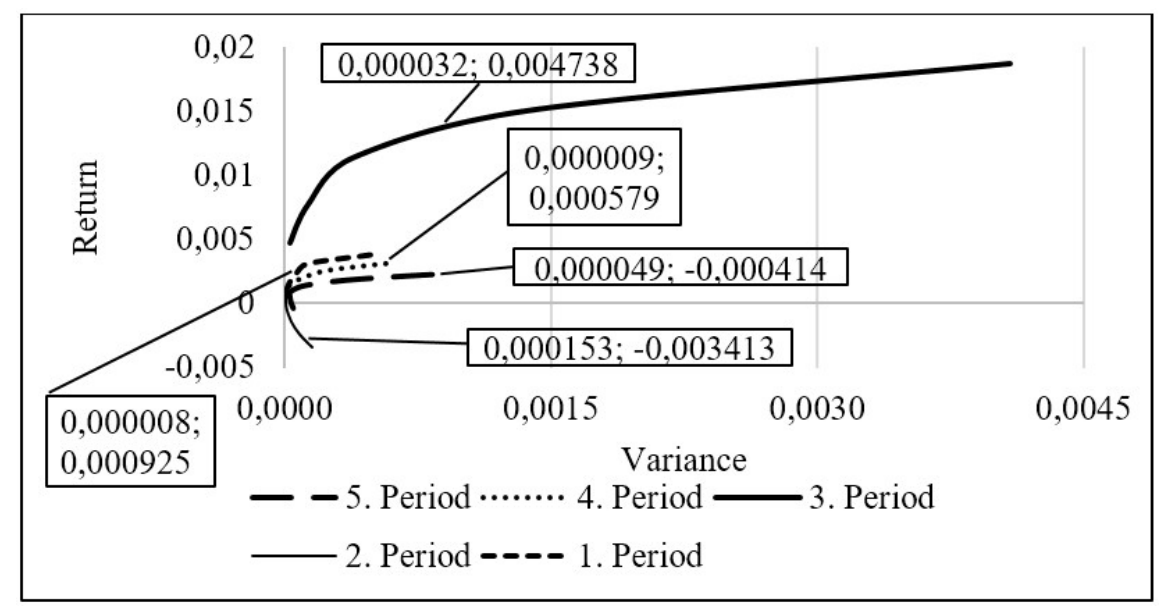

Figure 4: Efficient frontier

comparison with those obtained via e-M4 as in Table 1. This result might be a pitfall if the fixed cost is considered since it incurs for each asset in the portfolio. Hence if the number of assets in the portfolio increases, also cost increases and profit decreases.

When transaction cost and tax were reflected upon the portfolios optimized via MVM, it was clear that such an application provided almost the same PCR values, except in the first period. In that period, $\mathrm{PCR}$ values of e-M4 became higher with the increase of the investment amount. In other words, it is more favorable to invest in a portfolio of which PCR value is higher for the same variance (risk) and with the same investment amount.

The first period is the time beginning of which there is not any particular event that may affect the environment. The range of its efficient frontier is the narrowest starts from 0,000008 for the average expected return $(0,000924833)$ and to 0,000511 for the maximum return $(0,003844)$ as in Figure 4. It can be assumed that the first period is more stable compared to the other periods. Therefore, it can be stated that e-M4 may provide better results compared to the MVM of Markowitz in a stable environment.

\section{Conclusion}

This paper aims to examine the effects of the environment, investment amounts, and costs on the portfolios under fuzziness. Verdegay and Werners FLP approaches that were applied to Konno-Yamazaki PO model were used in this study. The constant linear structure of the tax cost with a piecewise constant linear structure of the transaction costs was integrated into these models as linear. The optimization was carried out with the models based on Verdegay and Werners FLP approaches, with their extended versions and with MVM of Markowitz.

When the optimized portfolios are considered in general, M4 can be suggested as a decisionmaking tool for risk-averse investors. Indeed, the objective function of this model is minimizing the risk. On the contrary, M6 can be suggested for risk taker-lover-investors. As a result, the Werners approach provides portfolios that include less number of assets by increasing the risk, whereas the Verdegay approach provides more extensive portfolios regarding the number of assets by distributing the risk.

The extended models may provide more realistic results compared to M4 and M6. To explain, even though M6 provides portfolios with a higher satisfaction/return by slightly increasing the risk, it can prevent investors from investing when the risk is high. Moreover, portfolios of the same period for different investment amounts differ from each other regarding the type, number and share of the assets. In other words, the investment amount directly affects 
portfolio variance and PCR. The costs also directly affect portfolios. The portfolios obtained via the extended models were utterly different from the portfolios acquired via M4 and M6 regarding the type, number and share of assets in the portfolio as well as the satisfaction level and the risks taken. On the other hand, when the extended models are compared with MVM of Markowitz, unfortunately, the results do not differ in an unstable environment but a stable environment. e-M4 provided better results compared to the MVM of PO results for the first investment period. On the other hand, e-M6 prevented investors from making any investment in an investment period. Therefore, it could not be compared with MVM of Markowitz. Also, it was clear that different portfolios for different investment amounts were obtained in the same period. Unfortunately, any particular generalization about the effect of the investment amount on the portfolio could not be made.

The main contributions of this paper are determining the effects of the investment amount, tax and transaction cost on portfolios, investigating the relationship between optimization models and investor types, considering various social, political and financial events, and examining if fuzziness can be a practical approach in PO in an unstable environment. Furthermore, we compare the optimization models with and without tax and transaction costs for different investment amounts. On the other hand, there are also some limitations. For example, lower and upper investment amounts, holding period, transactions with credit, short selling operations, and sell-out costs can be considered. The time intervals can be extended; the pool of assets may include risk-free assets or assets traded at foreign stock exchanges. Also, since none of the fuzzy models in this paper could provide better results than the MVM of Markowitz in an unstable environment (but produced better results in a stable environment), robust optimization can be considered where social, political or financial instability and so uncertainty is high by adopting different uncertainty set for social, political and financial indicators.

\section{Appendix}

Mean-Variance Model of Markowitz. Based on the Markowitz's approach to portfolio selection, an investor may have two objectives which are maximizing the portfolio return with $m_{x}=E R_{x}$ where $m_{x}$ denotes the average return of and $E R_{x}$ denotes the random return of the portfolio $x$, and minimizing the portfolio risk which is measured by $\sigma_{x}^{2}=\operatorname{Var} R_{x}$ or $\sigma_{x}$ where $\sigma_{x}$ denotes the standard deviation of the random variable $R_{x}$. The more "variable" the random return $R_{x}$ on the portfolio x, the higher the variance of $R_{x}$. If the return $R_{x}$ is certain then the variance is equal to zero, and so such a portfolio becomes risk-free. Therefore, an investor puts weights on these two conflicting objectives and wants to maximize $\tau m_{x}-\sigma_{x}^{2}$ where $\tau$ denotes the risk tolerance [6]. For more detailed information regarding the MVM of Markowitz, please refer to [6, 23].

Model 7:

$$
\max _{x \in \mathbb{R}^{N}}\left\{\tau \sum_{i=1}^{N} x_{i} m_{i}-\sum_{i=1}^{N} \sum_{j=1}^{N} \sigma_{i j} X_{i} X_{j}\right\}
$$

subject to

$$
\begin{aligned}
\sum_{i=1}^{N} X_{i} & =1 \\
X_{i} \geq 0 \text { for } i & =1, \ldots, N \\
\tau & >0
\end{aligned}
$$

Fixed cost. It is also one of the most common cost types incurs, especially when a broker manages a portfolio for an investor. For integrating the fixed cost to the model, a binary variable $\omega_{j}$ can be used as below: 


$$
\begin{aligned}
M_{R} \sum_{j=1}^{n}\left(r_{j} x_{j}\right)-C_{T X}-\sum_{j=1}^{n} f_{j} \omega_{j} & \geq M_{R}\left(\rho M_{0}+\alpha \tau\right)+C_{T R}, \alpha \in[0,1], \omega \in 0,1 \\
x_{j} & \leq \omega_{j} \\
\omega_{j} & =0 \text { or } 1
\end{aligned}
$$

where $f_{j}$ denotes the fixed cost in terms of currency per asset in the optimum portfolio and $\omega_{j}$ is a binary variable. If the share of the $j^{t h}$ asset is greater than zero, Eq. (36) forces $\omega_{j}=1$. On the contrary, if the share of the $j^{\text {th }}$ asset is zero, the desired value of $\omega_{j}$ becomes zero, and Eq. (36) does not guarantee it. In this case, it can be either zero or one. However, it does not prevent obtaining an optimum portfolio. To explain, assume an ideal solution, which is $x_{m}=0, \omega_{m}=0$, and left-hand side of Eq. (35) has a real value R, which is higher than the right-hand side of the Eq. (35). On the other hand, the other solution is $x_{m}=0, \omega_{m}=1$, and the left-hand side of Eq. (35) also has a real value $G$, which is also higher than the right-hand side of the Eq. (35). When these two values are compared, it is clear that $G<R$ because the higher fixed cost is subtracted from the return value on the left-hand side of the Eq. (35) due to $\omega_{m}=1$. It means that even if $\omega_{m}=1$ when $x_{m}=1$, the solution is still feasible for the same objective function.

Investment amount variable. If the investment amount is considered as a decision variable, the optimization model is transformed into a structure that requires the use of quadratic programming approach since Eq. (31) of the extended models become a quadratic constraint. The concave or convex structure of the cost functions should be examined first to determine the optimum investment amount. Then the equations of the functions should be generated. Finally, they are integrated into e-M4 and e-M6. However, the inclusion of the cost functions requires (0-1) type binary decision variables, and the model becomes a Mixed Integer Linear Programming model. For example, one of the most common transaction cost function type is a piecewise linear concave function. In this case, Eq. (37-43) may be considered additionally.

$$
\begin{gathered}
F\left(M_{R}\right)=z_{1} f\left(b_{1}\right)+z_{2} f\left(b_{2}\right)+\cdots+z_{n} f\left(b_{n}\right) \\
b_{n} \leq M_{R} \leq b_{n+1} \\
M_{R}=z_{1} b_{1}+z_{2} b_{2}+\cdots+z_{n} b_{n} \\
z_{1} \leq l_{1}, z_{2} \leq l_{1}+l_{2}, \cdots z_{n-1} \leq l_{n-2}+l_{n-1}, z_{n} \leq l_{n-1} \\
l_{1}+l_{2}+\cdots+l_{n-1}=1, l \in\{0,1\} \\
z_{1}+z_{2}+\cdots+z_{n}=1 \\
z_{n} \geq 0, b_{n} \geq 0
\end{gathered}
$$

where $M_{R}$ represents the real investment amount. $n$ represents the number of divides in costs. The starting and ending points of all line segments are symbolized by $b_{n}$ and it is known as the breaking point. Each line segment has been represented by $l_{n-1}$. This is a variable that ensures that the investment amount is located on the related line and does not take any value other than 0,1 which adds the related transaction cost in the model. $z_{n}$ corresponds to the line segment interval on which $M_{R}$, the investment amount is located. It has a value which yields the investment amount $M_{R}$ when the start and endpoint of the line segment are multiplied by $b_{n}$ and summed up. Thus, $z_{n}$ is a point on the line segment and it is the proportional location of the point on the line. In light of this information, only a single $l_{n-1}$ variable can have a value greater than zero at any instant, and this value can only be 1 . Only two $z_{n}$ variables can take on a value that is greater than zero due to the expressions $z_{1} \leq l_{1}, z_{2} \leq l_{1}+l_{2}, \ldots$, $z_{n-1} \leq l_{n-2}+l_{n-1}, z_{n} \leq l_{n-1}$. Thus, the investment amount $M_{R}$ is expressed in terms of $z_{n}$ 
and $b_{n}$. This structure may help to determine an optimum investment amount. To check if this formulation works please go back to Figure 3(a). Each line segment in it can be represented by $l_{n-1}$. The first line segment is defined by $l_{1}$ and $l_{2}$ is defined as the second line segment, etc. For the optimization problem in this paper, there are 6 line segments or investment intervals. As an example, $l_{1}=1$ indicates that the investment amount $M_{R}$ is between the values of $(0-50,000)$ TL and that all other $l_{n-1}$ are zero because $l_{1}+l_{2}+\cdots+l_{n-1}=1, l \in\{0,1\}$. After making all necessary calculations, the additional equations are finalized as follows.

$$
\begin{gathered}
f\left(b_{n}\right)=\{0,75,140,305,530,880, \text { and } 1380\} \\
F\left(M_{R}\right)=z_{1} 0+z_{2} 75+z_{3} 140+z_{4} 305+z_{5} 530+z_{6} 880+z_{7} 1380 \\
M_{R}=z_{1} 0+z_{2} 50000+z_{3} 100000+z_{4} 250000+z_{5} 500000+z_{6} 10^{6}+z_{7} 2 e^{6} \\
z_{1} \leq l_{1}, z_{2} \leq l_{1}+l_{2}, z_{3} \leq l_{2}+l_{3}, z_{4} \leq l_{3}+l_{4}, z_{5} \leq l_{4}+l_{5}, z_{6} \leq l_{5}+l_{6}, z_{7} \leq l_{6} \\
l_{1}+l_{2}+\cdots+l_{6}=1, l \in\{0,1\} \\
z_{1}+z_{2}+\cdots+z_{7}=1 \\
z_{n} \geq 0, b_{n} \geq 0 n=1, \ldots, 7
\end{gathered}
$$

Let us assume $M_{R}=90,000 \mathrm{TL}$ as the optimum investment amount. This amount is located in the interval of $50000 \leq M_{R} \leq 100000$ and is located on the line segment $l_{2}$. Thus, $l_{2}=1$ and all other $l_{n-1}=0$ and the variable $l_{2}$ is included only in the $z_{2} \leq l_{1}+l_{2}, z_{3} \leq l_{2}+l_{3}$ inequalities. Therefore, $z_{2} \neq 0$ and $z_{3} \neq 0$. The start and endpoints of this line segment are $\left(b_{1}-b_{2}\right)=(50,000-100,000)$. In the light of these findings, Eq. (39) and Eq. (42) are rearranged and the values of $z_{2}$ and $z_{3}$ are determined as below.

$$
\begin{aligned}
50000 z_{2}+100000 z_{3} & =90000 \\
z_{2}+z_{3} & =1 \\
& \Rightarrow z_{2}=\frac{1}{5} \text { and } z_{3}=\frac{4}{5}
\end{aligned}
$$

Then these values are placed in Eq. (37) and the transaction cost for 90,000 TL is calculated as $F(90,000)=75 z_{2}+140 z_{3}=127$ TL.

M4, M6, e-M4 and e-M6 become mixed-integer linear programming models after the inclusion of the fixed cost Eq. (35, 36), and investment amount Eq. (37-43).

\section{References}

[1] Arnott, R. and Wagner, W. (1990). The measurement and control of trading costs. Financial Analyst Journal, 46: 73-80. www.jstor.org/stable/4479381

[2] Chang, Ching T. (2005). A modified goal programming approach for the mean-absolute deviation portfolio optimization model. Applied Mathematics and Computation, 171(1), 567-572. doi:10.1016/j.amc.2005.01.072

[3] CNN Business. Thompson, M. (2014). Worst is yet to come for fragile five. https://money.cnn.com/2014/01/30/investing/emerging-markets-fragile-five/index.html [Accessed 27/05/19]

[4] CNN International. Hakura, F. (2014). Is instability the 'new normal' for turkey? https://edition.cnn.com/2014/01/08/opinion/turkey-politics-conservatism-hakura/index.html [Accessed 27/05/19]

[5] CNN International. Kottasova, I. (2014). Turkish economic mess: how did it get to this point? https://edition.cnn.com/2014/03/28/business/turkey-economy-election/index.html [Accessed 27/05/19] 
[6] Evstigneev, Igor V., Hense, T. and Schenk-Hoppé, K. R. (2015). Mean-variance portfolio analysis: The markowitz model. In Evstigneev, Igor V., Hense, T. and Schenk-Hoppé, K. R. (Eds.). Mathematical Financial Economics: A Basic Introduction, 11-19, Switzerland: Springer. doi:10.1007/978-3-319-16571-4_2

[7] Fang, Y., Lai, K. K. and Wang, S. Y. (2006). Portfolio rebalancing model with transaction costs based on fuzzy decision theory. European Journal of Operational Research, 175(2), 879--93. doi:10.1016/j.ejor.2005.05.020

[8] Fang, Y., Lai, K. K. and Wang, S. Y. (2008). Fuzzy Portfolio Optimization. Berlin: Springer. doi:10.1007/978-3-540-77926-1

[9] Filomena, T. P. and Lejeune, M. A. (2014). Warm-start heuristic for stochastic portfolio optimization with fixed and proportional transaction costs. Journal of Optimization Theory and Applications, 161(1): 308--29. doi:10.1007/s10957-013-0348-y

[10] Kellerer, H., Mansini, R. and Speranza, M. G. (2000). Selecting portfolios with fixed costs and minimum transaction lots. Annals of Operations Research, 99(1): 287--304. doi:10.1023/A:1019279918596

[11] Kocadağlı, O. and Cinemre, N. (2010). A fuzzy non-linear model approach with CAPM for portfolio optimization. Istanbul University Journal of the School of Business Administration, 39(2): 359--69.

[12] Konno, H. and H. Yamazaki. (1991). Mean-absolute deviation portfolio optimization model and its applications to Tokyo stock market. Management Science, 37(May): 519--31. https://www.jstor.org/stable/2632458

[13] Konno, H. and Wijayanayake, A. (1999). Mean-absolute deviation portfolio optimization model under transaction costs. Journal of the Operations Research Society of Japan, 42(4): 422-35. http://www.orsj.or.jp/ archive/pdf/e_mag/Vol.42_04_422.pdf [Accessed 1/12/19]

[14] Konno, H. and Yamamoto, R. (2003). Minimal concave cost rebalance of a portfolio to the efficient frontier. Mathematical Programming, Series B, 97(3): 571--85. doi:10.1007/s10107-003-0428-0

[15] Konno, H. and Yamamoto, R. (2005). Global optimization versus integer programming in portfolio optimization under nonconvex transaction costs. Journal of Global Optimization, 32(2): 207--19. doi:10.1007/s10898-004-2703-x

[16] Lai, Y. J. and Hwang, C. L. (1992). Fuzzy Mathematical Programming. Berlin: Springer. doi:10.1007/978-3-642-48753-8

[17] Leon, T., Liern,V. and Vercher, E. (2000). Fuzzy mathematical programming for portfolio management. In: Bonilla M., Casasús T. and Sala R. (Eds.). Financial Modelling. Contributions to Management Science, 241--56. Berlin: Springer. doi:10.1007/978-3-642-57652-2_17

[18] Li, X., Zhang, Y., Wong, H. S. and Qin, Z. (2009). A hybrid intelligent algorithm for portfolio selection problem with fuzzy returns. Journal of Computational and Applied Mathematics, 233(2): 264-78. doi:10.1016/j.cam.2009.07.019

[19] Liagkouras, K. and Metaxiotis, K. (2018). Multi-period mean-variance fuzzy portfolio optimization model with transaction costs. Engineering Applications of Artificial Intelligence, 67(2018): 260-269. doi:10.1016/j.engappai.2017.10.010

[20] Liu, Y. J., Zhang, W. G and Zhao, X. J. (2016). Fuzzy multi-period portfolio selection model with discounted transaction costs. Soft Computing, 22(1): 177-193. doi:10.1007/s00500-016-2325-5

[21] Lobo, M. S., Fazel, M. and Boyd, S. (2007). Portfolio optimization with linear and fixed transaction costs. Annals of Operations Research, 152(1): 341--65. doi:10.1007/s10479-006-0145-1

[22] Mansini, R., Ogryczak, W. and Speranza, M. G. (2014). Twenty years of linear programming based portfolio optimization. European Journal of Operational Research, 234(2): 518--35. doi:10.1016/j.ejor.2013.08.035

[23] Markowitz, H. (1952). Portfolio Selection. The Journal of Finance, 7(1): 77--91. doi:10.1111/j.1540-6261.1952.tb01525.x

[24] Mei, X, DeMiguel, V. and Nogales, F. J. (2016). Multiperiod portfolio optimization with multiple risky assets and general transaction costs. Journal of Banking and Finance, 69(2016):108-120. doi:10.1016/j.jbankfin.2016.04.002

[25] Olivares-Nadal, A. V. and DeMiguel, V., (2018). A robust perspective on transaction costs in portfolio optimization. Operations Research, 66(3): 733-739. doi:10.1287/opre.2017.1699

[26] Östermark, R. (1996). A fuzzy control model (fcm) for dynamic portfolio management. Fuzzy Sets 
and Systems, 78(3): 243--54. doi:10.1016/0165-0114(96)84605-7

[27] Pachamanova, D. A. and Fabozzi, F. J. (2010). Simulation and Optimization in Finance. New Jersey: John Wiley\&Sons, Inc.

[28] Ramaswamy, S. (1998). Portfolio selection using fuzzy decision theory. Bank for International Settlements. Working Paper No 59, Basle. https://ideas.repec.org/p/bis/biswps/59.html

[29] Rommelfanger, H. (1996). Fuzzy linear programming and applications. European Journal of Operational Research, 92(3): 512-527. doi:10.1016/0377-2217(95)00008-9

[30] Tanaka, H. and Guo, P. (1999). Portfolio selection based on upper and lower exponential possibility distributions. European Journal of Operational Research, 114(1): 115--26. doi:10.1016/S03772217(98)00033-2

[31] Thi, H. A. L., Moeini, M. and Dinh, T. P. (2009). DC programming approach for portfolio optimization under step increasing transaction costs. Optimization, 58(3): 267-289. doi:10.1080/02331930902741721

[32] Verdegay, J. L. (1984). A dual approach to solve the fuzzy linear-programming problem. Fuzzy Sets and Systems, 14(2): 131-141. doi:10.1016/0165-0114(84)90096-4

[33] Watada, J. (2001). Fuzzy portfolio model for decision making in investment. In Yoshida, Y. (Eds.). Dynamical Aspects in Fuzzy Decision Making, 141-162. Berlin: Springer . doi:10.1007/978-3-79081817-8_7

[34] Werners, B. (1987). An interactive fuzzy programming system. Fuzzy Sets and Systems, 23(1): 131-147. doi:10.1016/0165-0114(87)90105-9

[35] Woodside-Oriakhi, M., Lucas, C. and Beasley, J. E. (2013). Portfolio rebalancing with an investment horizon and transaction costs. Omega, 41: 406-420. doi:10.1016/j.omega.2012.03.003

[36] World Finance. Herdem, S. (2014). On the move: Turkey's foreign direct investment market. https://www.worldfinance.com/strategy/turkeys-foreign-direct-investment-market [Accessed $27 / 05 / 19]$

[37] Young, M.R. (1998). A minimax portfolio selection rule with linear programming solution. Management Science, 44: 673-683. https://www.jstor.org/stable/2634472

[38] Zadeh, L. A. (1965). Fuzzy sets. Information and Control, 8(3): 338-353. doi:10.1016/S00199958(65)90241-X

[39] Zimmermann, H. J. (2001). Fuzzy Set Theory and Its Applications. Berlin: Springer. 